\title{
Leptospirose-assoziiertes Kawasaki-Syndrom - Differenzialdiagnose oder Krankheitsassoziation?
}

Das Kawasaki-Syndrom (KS) ist eine systemische Vaskulitis, die mittelgroße Arterien betrifft. Die genaue Pathophysiologie ist nicht bekannt [1]. Neben der genetischen Veranlagung werden immunologische und infektiöse Auslöser berichtet [2-4]. Darüber hinaus wurden in der jüngsten SARSCOV-2-Pandemie mehrere Fälle von pädiatrischen Patienten gemeldet, bei denen ein KS oder eine Hyperinflammation im Zusammenhang mit einem pädiatrisch entzündlichen Multisystem-Syndrom (PIMS) auftritt [5-8].

Das KS sollte hauptsächlich bei Vorschulkindern in Betracht gezogen werden, die Fieber, Bindehautentzündungen, Lymphadenitiden, oropharyngeale Schleimhautentzündungen, palmoplantare Schwellungen und generalisierte Hautausschläge aufweisen [2, 9]. Die Patienten können in der zweiten Woche eine periunguale Schuppung an Fingern und Zehen entwickeln, ein relativ spezifisches Kennzeichen eines KS [10]. Die richtige Diagnose kann schwierig sein, da kein Bestätigungstest vorliegt.

In diesem Artikel stellen wir den Fall einer 3-jährigen Patientin vor, bei der ein komplettes KS diagnostiziert wurde, einschließlich einer periungualen Schuppung der Finger. Dieses Mädchen wurde auch positiv auf Leptospiren getestet.

\section{Falldarstellung}

Eine 3-jährige Patientin mit Fieber seit 4 Tagen wurde in unser Krankenhaus eingeliefert. Sie hatte eine zervikale Lymphadenitis, eine bilaterale Bindehautentzündung und entwickelte einen erythematösen Hautausschlag, hauptsächlich am Rumpf ( $\triangleright$ Abb. 1). Sie konnte wegen Arthralgien und Myalgien nicht laufen. Sie hatte leichten Durchfall ohne Erbrechen. Während der Untersuchung wurde eine Erdbeerzunge sowie eine Palmar- und Plantarschwellung festgestellt. Es wurden keine Auffälligkeiten an anderen Organsystemen entdeckt.

Die Mutter gab an, dass es Mäuse in ihrem Haus gäbe. Die Blutuntersuchung ergab erhöhte Entzündungsparameter: CRP:

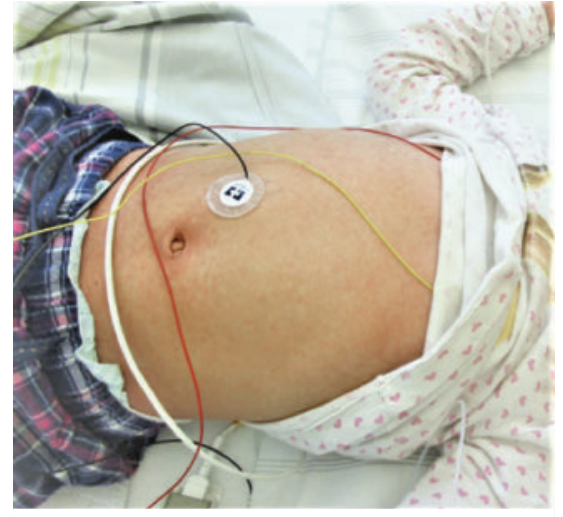

- Abb. 1 Erythematöser Hautausschlag.

$12,4 \mathrm{mg} / \mathrm{dl}(<0,5)$; BSG: $90 \mathrm{~mm} / \mathrm{h}(<20)$; Leukozyten: $5,2 \times 10^{3} / \mu \mathrm{l}\left(5,5-15 \times 10^{3} /\right.$ $\mu \mathrm{l})$; Thrombozyten: $313 \times 10^{3} / \mu \mathrm{l}(210-$ $\left.500 \times 10^{3} / \mu \mathrm{l}\right)$; AST: $105 \mathrm{U} / \mathrm{I}(<60) ; \mathrm{ALT}$ : $105 \mathrm{U} / \mathrm{l}(<35)$; NT-ProBNP: $991 \mathrm{pg} / \mathrm{ml}$ $(<400)$. Der Urin zeigte eine leichte Proteinurie (Protein/Kreatinin-Ratio: $0,9 \mathrm{~g} / \mathrm{g}$ Kreatinin). Serologische Tests auf Epstein-Barr-Virus, Adenovirus, Puumalavirus, Masern und COVID-19 waren negativ.

Da die Patientin alle Kriterien für ein komplettes KS erfüllte, wurden Immunglobuline $2 \mathrm{~g} / \mathrm{kg}$ und ASS $50 \mathrm{mg} / \mathrm{kg} /$ Tag gestartet. Da die Patientin klinisch schwer krank war, hohe Entzündungsparameter sowie einen hohen NT-Pro-BNP-Wert aufwies, wurde additiv mit Prednisolon $2 \mathrm{mg} / \mathrm{kg} / \mathrm{Tag}$ begonnen. In weniger als 24 Stunden sistierte das Fieber und die Patientin besserte sich klinisch. Die Echokardiografie zeigte keine Anzeichen einer Herzbeteiligung. Die Entzündungsparameter nahmen ab und zeigten am Tag 7 des Krankheitsverlaufs ein normales CRP.

Aufgrund des Berichts der Mutter über den Mauskontakt und der beschriebenen Symptome in Kombination mit der Proteinurie wurde eine Diagnostik auf Leptospirose (PCR im Urin und Leptospirose-Antikörper im Blut) veranlasst. Die Urin-PCR war positiv, sodass -bei ausgeprägtem Krankheitsgefühl - eine additive antibiotische Therapie mit Penicillin begonnen wurde. Die spezifischen Leptospirose-Antikörper im Serum waren negativ. In der 2. Woche der Krankheit entwickelte die Patientin

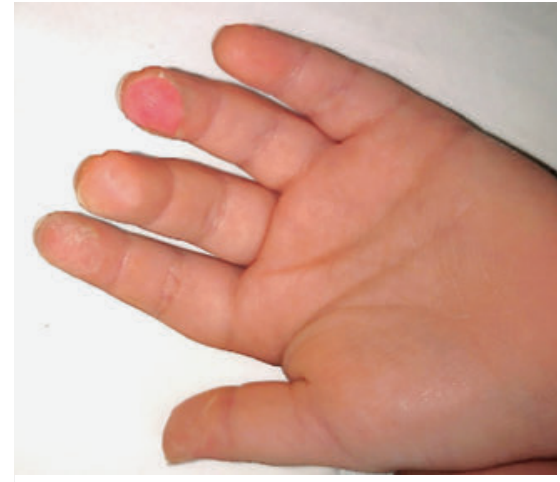

- Abb. 2 Periunugale Schuppung im Bereich der Fingerkuppen.

eine deutliche periunguale Fingerschuppung ( $\triangleright$ Abb. 2). Bei der Nachuntersuchung nach 6 Wochen blieb die Patientin asymptomatisch und zeigte echokardiografisch keine Koronaraneurysmata oder andere KS-assoziierte Komplikationen wie Hörstörungen.

\section{ZUSAMMENFASSUNG}

Einleitung: Das Kawasaki-Syndrom (KS) ist eine systemische Vaskulitis, die mittelgroße Arterien betrifft. Obwohl Diagnose- und Klassifizierungskriterien existieren, kann die Abgrenzung von anderen Krankheiten schwierig sein.

Falldarstellung: Bei einer 3-jährigen Patientin wurden neben Fieber folgende Symptome und Befunde festgestellt: Konjunktivitis, zervikale Lymphadenopathie, Palmarschwellung, gerötete und rissige Lippen sowie ein makulöses Exanthem. Diese Befunde sind mit einem kompletten KS vereinbar. Die Urin-PCR auf Leptospiren war positiv. Nach 2 Wochen entwickelte sich eine periunguale Schuppung.

Diskussion: Dieser Fall zeigt, dass eine Leptospirose nicht nur ähnliche Symptome wie das KS aufweist, sondern auch mit dieser Erkrankung assoziiert sein kann. Dafür sprechen spezifische Befunde wie Schuppungen an Fingern, Zehen und Veränderungen der oropharyngealen Schleimhäute. 


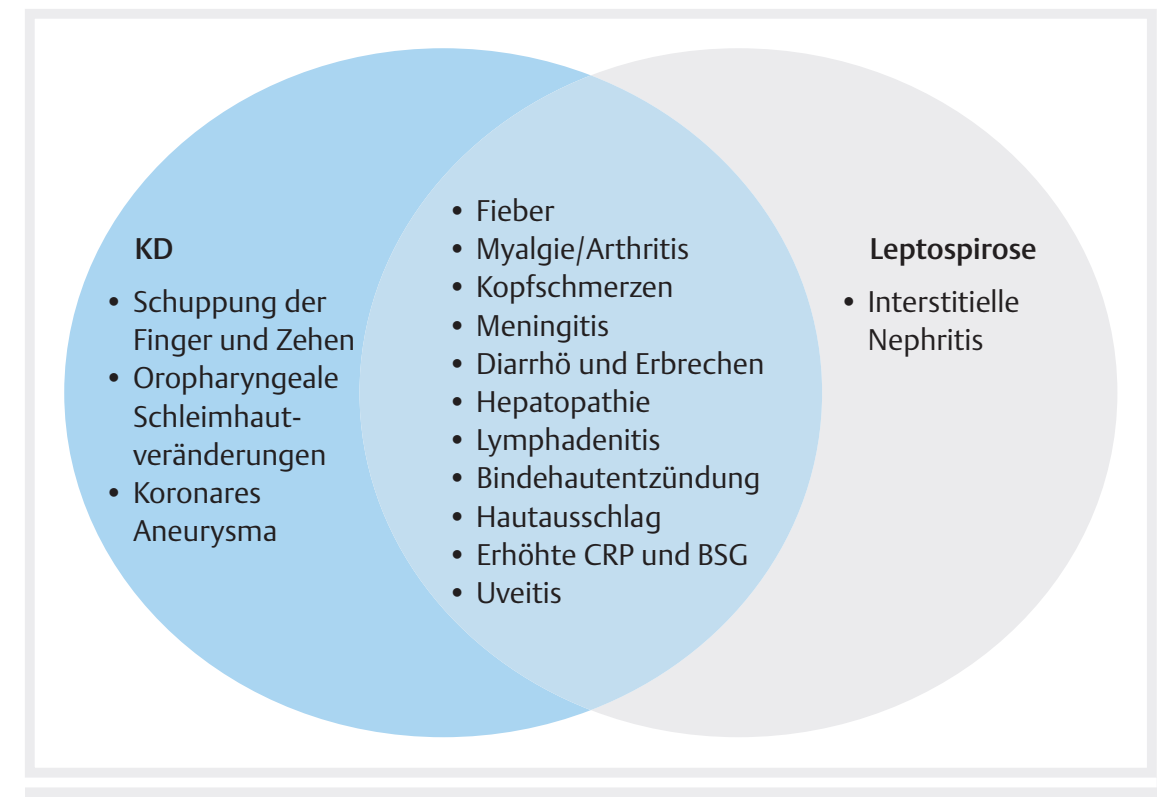

- Abb. 3 Vergleich zwischen Befunden und Symptomen beim KS und bei Leptospirose.

\section{Diskussion}

Die Leptospirose ist eine Zoonose, die durch ein Spirochätenbakterium verursacht wird, das im Urin verschiedener Tiere wie Hunde, Nutztiere und Nagetiere ausgeschieden wird. Die Übertragung erfolgt meist durch Kontakt mit kontaminiertem Urin [11].

Obwohl sich Schätzungen zufolge mehr als eine Million Menschen weltweit infizieren, sind weniger als 20 pädiatrische Fälle pro Jahr berichtet [14]. Eine Antibiotikabehandlung wird insbesondere bei schweren Verläufen empfohlen.

Die rasche Verbreitung des Erregers führt zum klinischen Bild eines septikämischen Stadiums von 3 bis 7 Tagen Dauer mit Fieber, Kopfschmerzen, Myalgien (40-97\%), Übelkeit, Konjunktivitis (28-99\%), begleitet oder gefolgt von einer immunvermittelten Phase (Dauer: 0 Tage bis 1 Monat) mit Meningitis, Uveitis, Hautausschlag und Fieber [11-13]. Eine Leptospireninfektion kann jedes Gewebe betreffen und neben einer hämorrhagischen Vaskulitis auch zu einer hepatozellulären Dysfunktion und einer Proteinurie führen. Diagnosebeweisend ist der Nachweis im Urin mittels PCR; negative serologische Ergebnisse - trotz bestätigter Diagnose - sind in der Literatur beschrieben $[11,13]$.
Da viele Befunde und Symptome zwischen einer Leptospirose und einem KS identisch sind, kann die Leptospirose sowohl imitierend als auch assoziativ mit einem KS auftreten ( $\mathbf{A} \mathbf{b b} \mathbf{3} \mathbf{3}$ ). Die periungualen Schuppungen und die oropharyngealen Schleimhautveränderungen und Koronaraneurysmen sind dabei ausschließlich für das KS berichtet $[2,11]$. Im vorliegenden Fall präsentierte die Patientin das klinische Bild eines kompletten KS; bekanntermaßen kann diese Diagnose auch beim Vorliegen nicht KD-typischer Kriterien wie nephritischen Urinveränderungen gestellt werden $[11,12]$.

\section{FAZIT}

Zusammenfassend gibt es eine breite klinische Überlappung zwischen KS und Leptospirose. Dabei sollte die Möglichkeit der Auslösung eines KS nicht übersehen werden, um mit einer frühzeitig eingeleiteten Therapie Folgeschäden vermeiden zu können. Eine Anamnese inklusive Reisen und Kontakt zu Tieren ist der erste Schritt zu einer solchen Diagnose.

\section{Interessenkonflikt}

Die Autorinnen/Autoren geben an, dass kein Interessenkonflikt besteht.
Einhaltung ethischer Richtlinien

Eine Einwilligung der Mutter der Patientin zur Publikation der Kasuistik liegt vor.

\section{Autorinnen/Autoren}

Mohammed Nashawi ${ }^{1}$
Friedrich Reichert $^{2}$
Friederike Blankenburg $^{1}$
Anita Heinkele $^{1}$
Christian Stirnkorb ${ }^{1}$
Felix Noll1
Kirsten Timmermann ${ }^{3}$
Toni Hospach ${ }^{1}$
${ }^{1}$ Zentrum für pädiatrische Rheumatologie
am Klinikum Stuttgart
${ }^{2}$ Abteilung für pädiatrische Infektiologie
und pädiatrische Notfallmedizin, Klinikum
Stuttgart
${ }^{3}$ Abteilung für Pädiatrische Nephrologie,
Klinikum Stuttgart

\section{Korrespondenzadresse}

Dr. Mohammed Nashawi

Zentrum für pädiatrische Rheumatologie am Klinikum Stuttgart Kriegsbergstraße 62, 70174 Stuttgart Deutschland

m.nashawi@klinikum-stuttgart.de

\section{Literatur}

[1] Hedrich CM, Schnabel A, Hospach T. Kawasaki disease. Frontiers in pediatrics 2018; 6: 198

[2] Modesti AM, Plewa MC. Kawasaki Disease. [Updated 2020 Jul 2]. In: StatPearls [Internet]. Treasure Island (FL): StatPearls Publishing 2020 Jan. Available from: https:// www.ncbi.nlm.nih.gov/books/NBK537163/

[3] Ramphul K, Mejias SG. Kawasaki disease: a comprehensive review. Arch Med Sci Atheroscler Dis 2018 Mar 21; 3: e41-e45. doi: 10.5114/amsad.2018.74522. PMID: 30775588; PMCID: PMC6374576

[4] Duignan S, Doyle SL, McMahon CJ. Refractory Kawasaki disease: diagnostic and management challenges. Pediatric health, medicine and therapeutics 2019; 10: 131-139. https://doi.org/10.2147/PHMT. S165935

[5] McCrindle BW, Rowley AH, Newburger JW et al. Diagnosis, Treatment, and LongTerm Management of Kawasaki Disease: A Scientific Statement for Health Profession- 
als from the American Heart Association. Circulation 2017; 135(17): e927-e999

[6] Pouletty M, Borocco C, Ouldali N et al. Paediatric multisystem inflammatory syndrome temporally associated with SARS-CoV-2 mimicking Kawasaki disease (Kawa-COVID-19): a multicentre cohort. Ann Rheum Dis 2020; 79(8): 999-1006

[7] Verdoni L, Mazza A, Gervasoni A et al. An outbreak of severe Kawasaki-like disease at the Italian epicentre of the SARS-CoV-2 epidemic: an observational cohort study. Lancet 2020; 395(10239): 1771-1778

[8] Viner RM, Whittaker E. Kawasaki-like disease: emerging complication during the COVID-19 pandemic. Lancet 2020; 395 (10239): 1741-1743

[9] Ozen S, Ruperto N, Dillon M et al. EULAR/ PReS endorsed consensus criteria for the classification of childhood vasculitides. Annals of the rheumatic diseases 2006; 65(7): 936-941

[10] Wang S, Best BM, Burns JC. Periungual desquamation in patients with Kawasaki disease. The Pediatric infectious disease journal 2009; 28(6): 538

[11] American Academy of Pediatrics. Leptospirosis. In: Kimberlin DW, Brady MT, Jackson
MA, Long SS, eds. USA: Red Book; 2018. 2018 Report of the Committee on Infectious Diseases. $31^{\text {st }}$ ed.

[12] De Vries SG, Bekedam MMI, Visser B] et al. Travel-related leptospirosis in the Netherlands 2009-2016: an epidemiological report and case series. Travel medicine and infectious disease 2018; 24: 44-50

[13] Shapiro ED, Leptospirosis. In: Long SS, Prober CG, Fischer M, eds. Principles and Practice of Pediatric Infectious Diseases. 5th ed. Philadelphia/PA, USA: Elsevier; 2018

[14] Robert Koch-Institut: SurvStat@RKI 2.0, https://survstat.rki.de, date of inquiry: 19.07.2020

\section{Bibliografie}

arthritis + rheuma 2021; 41: 148-150

DOI 10.1055/a-1395-9115

ISSN $0176-5167$

(c) 2021. Thieme. All rights reserved. Georg Thieme Verlag KG, Rüdigerstraße 14, 70469 Stuttgart, Germany 